\title{
Discrimination method of direction of arrival estimation correctness based on deep neural network
}

\author{
Ryusuke Tanaka* and Yoichi Haneda ${ }^{\dagger}$ \\ Informatics and Engineering, Graduate School of University of Electro-Communications, \\ 1-5-1 Chofugaoka, Chofu, 182-0021 Japan
}

(Received 30 September 2018, Accepted for publication 18 May 2019)

Keywords: DOA estimation, Reverberation, Noise, Deep neural network, Supervised learning PACS number: 43.60.+d [doi:10.1250/ast.41.318]

\section{Introduction}

We herein propose a discrimination method based on deep neural network (DNN) [1] to determine whether an estimated direction of arrival (DOA) is correct or an error for DOA estimation using a microphone array. DOA estimation is an important technique for video conferencing, speech recognition systems, and so forth. Because the accuracy of the estimated DOA is degraded by interference signals such as noise and reverberation, many DOA estimation methods have been developed over the last few decades for real environments [2,3]. In recent years, several supervised learning methods using a DNN have been proposed.

A problem in DOA estimation methods that have been studied is that the extent of the error of the estimated DOA for a microphone array system is not known. If we can determine whether the estimated DOA is correct or an error, then the estimation accuracy can be improved. For example, if the estimated DOA is an error, by rejecting this estimation and estimating the DOA from another signal, the accuracy will be improved. Herein, we call this process a true/false (TF) discrimination. To realize TF discrimination, we propose a learning-based method that utilizes a DNN to learn TF discrimination and DOA estimation simultaneously. TF discrimination is learned by an objective function based on the error between the outputs of the DNN and the true DOA. This objective function is designed to reflect whether the estimated DOA of the DNN has been corrected. The proposed method is validated by computer simulations and experimental results.

\section{DOA estimation with DNN}

A DNN has a multilayered architecture whose layers transform the inputs using a nonlinear function and an affine transform. Each layer of the DNN comprises $L$ layers of weight $\boldsymbol{W}^{(l)}$ and bias $\boldsymbol{b}^{(l)}(l=1, \ldots, L-1)$. A nonlinear transformation is conducted using the following equation:

$$
\boldsymbol{x}^{(l+1)}=f^{(l)}\left(\boldsymbol{W}^{(l)} \boldsymbol{x}^{(l)}+\boldsymbol{b}^{(l)}\right),
$$

where $\boldsymbol{x}^{(l+1)}$ represents the $l$ th layer output; in particular, $\boldsymbol{x}^{(1)}$ represents the input features and $\boldsymbol{x}^{(L)}$ represents the DNN output. $f^{(l)}$ represents the activation function, which is a nonlinear function such as the sigmoid function or the

\footnotetext{
*e-mail: t1840004@edu.uec.ac.jp

†e-mail: haneda.yoichi@uec.ac.jp
}

rectified linear unit (ReLU) [4]. In the multilabel classification problem, the softmax function is used for $f^{(L-1)}$. The DNN for multilabel classification is learned by minimizing the cross entropy function as follows:

$$
J_{\mathrm{CE}}\left(\boldsymbol{x}^{(1)}\right)=\mathbb{E}\left[\sum_{m=1}^{M} x_{m}^{\text {true }} \log x_{m}^{(L)}\right],
$$

where $M$ is the number of labels and the subscript represents the $i$ th vector elements. $\mathbb{E}[\cdot]$ is the expectation. $x_{m}^{\text {true }}$ is the true probability of the $m$ th label.

In the learning process of DOA estimation, labels are assigned to the DOAs. For the input features, the observed signals of a microphone array or other features that include DOA information are used. The DNN can facilitate DOA estimation with a pair of input features and true DOAs using supervised learning.

DOA estimation methods based on supervised learning have been investigated [5-7]. An advantage of learning-based methods is that the estimation performance can be improved without modeling the interference. Various learning models have been investigated to improve the estimation accuracy in real environments. However, similar to the model-based DOA estimation methods, the approach of discriminating whether the estimated DOA for an unknown data is correct or is an error has not been considered.

\section{Proposed method}

This section details the proposed method. This method consists of two primary aspects. First, the DNN architecture for TF discrimination is considered. Next, the objective function for learning $\mathrm{TF}$ discrimination is designed to be minimized if the correctness of the estimated DOA is discriminated accurately. The estimation accuracy can be improved by adopting only the DOA that is discriminated as correct by the TF discrimination DNN.

\subsection{Architecture of DNN}

TF discrimination is conducted with a DNN. We herein investigate two architectures for the DNN. In one architecture, the intermediate output of the DOA estimation DNN is used as the input features for the TF discrimination DNN. Figure 1 shows a diagram of the proposed DNN architecture when using the intermediate output of the DNN, the input features for TF discrimination would include information on DOA estimation DNN. Layer one, which is located before the output layer, is used as the input layer of the TF discrim- 


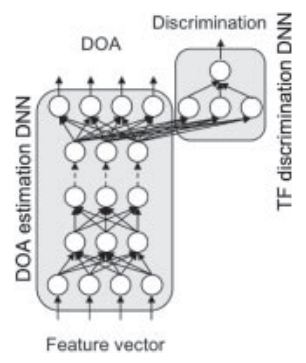

Fig. 1 Diagram of proposed DNN architecture using the intermediatelayer outputs as the input features for TF discrimination DNN.

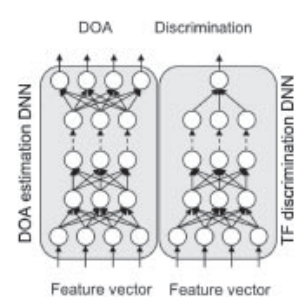

Fig. 2 Diagram of proposed DNN architecture using the same feature vector for both TF discrimination DNN and DOA estimation DNN.

ination DNN in this study. The layer location of the TF discrimination DNN is validated in the experiments reported in Sect. 4. In the other architecture, the TF discrimination DNN and DOA estimation DNN are independent of each other. Figure 2 shows a diagram of second architecture.

Because TF discrimination is a binary classification, the sigmoid function is used for the activation function of the output layer of the DNN. The output of the TF discrimination DNN is expressed as $p\left(\boldsymbol{x}^{(1)}\right)$, where $\boldsymbol{x}^{(1)}$ indicates the input features.

3.2. Objective function for learning TF-discrimination

A new criterion is required to learn TF discrimination because supervised data for TF discrimination do not exist. The TF discrimination DNN learns from the estimation error of the DOA estimation DNN in this study. For inputs where the DNN outputs large errors of the DOA even if the learning converges, the TF discrimination DNN learns to output the label which indicates the estimated DOA is erroneous. For inputs where the DNN outputs a small error of the DOA, the TF discrimination DNN learns to output the label which indicates the estimated DOA is correct.

In this study, the labels of "correct" or "error" follow the Bernoulli distribution [8], exhibits a binary value for random variables. The Bernoulli distribution is assumed to be the probability distribution for a binary classification problem when learning a binary classification model. By employing a probabilistic model, we can design the objective function analytically. The probability mass function $P(k)$ of the Bernoulli distribution is expressed as the following equation:

$$
P(k)=q^{k}(1-q)^{1-k}
$$

where $q$ is the probability that the output is correct, $k=0,1$ is the label index, which indicates correct estimation when $k=$ 1 and an error when $k=0$. In the binary classification problem, the probability $q$ is estimated for the input features. Therefore, $p\left(\boldsymbol{x}^{(1)}\right)$ is regarded as the probability that the estimated DOA is correct because $p\left(x^{(1)}\right) \in[0,1]$.

The objective function of the output of the DOA estimation DNN for $\boldsymbol{x}^{(1)}$ is written as $J_{\mathrm{DOA}}\left(\boldsymbol{x}^{(1)}\right)$. Instead of the supervised data for TF discrimination learning, we use the objective function $J_{\mathrm{TF}}\left(\boldsymbol{x}^{(1)}\right)$ based on $J_{\mathrm{DOA}}\left(\boldsymbol{x}^{(1)}\right)$ for the estimation of TF discrimination.

This objective function is $J_{\mathrm{DOA}}\left(\boldsymbol{x}^{(1)}\right)$ if the TF discrimination DNN output label as the estimated DOA is correct. The objective function $J_{\mathrm{TF}}\left(\boldsymbol{x}^{(1)}\right)$ is the constant $\lambda_{\mathrm{p}}$ if the TF discrimination DNN output label as the estimated DOA is an error. $\lambda_{\mathrm{p}}$ is set by the user. If $J_{\mathrm{DOA}}\left(\boldsymbol{x}^{(1)}\right)$ is larger than $\lambda_{\mathrm{p}}$, the TF-discrimination-DNN must output the estimated DOA as an error to minimize the objective function, i.e., the DNN outputs a small value of $p\left(\boldsymbol{x}^{(1)}\right)$. Conversely, if $J_{\mathrm{DOA}}\left(\boldsymbol{x}^{(1)}\right)$ is smaller than $\lambda_{\mathrm{p}}$, the TF-discrimination-DNN must output the estimated DOA as correct to minimize the objective function, i.e., the DNN outputs a small value $p\left(\boldsymbol{x}^{(1)}\right)$. Therefore, by minimizing the objective function, TF discrimination can be learned without supervised data.

Because $p\left(\boldsymbol{x}^{(1)}\right)$ follows the Bernoulli distribution, the expectation value can be written as the following equation;

$$
\mathbb{E}\left[J_{\mathrm{TF}}\right]=J_{\mathrm{DOA}} p+\lambda_{\mathrm{p}}(1-p) .
$$

$\boldsymbol{x}^{(1)}$ is omitted from the previous equation. In this study, the proposed DNN learning originates from the following objective function:

$$
J=\frac{1}{I} \sum_{i=1}^{I}\left(J_{\mathrm{TF}}\left(\boldsymbol{x}_{i}^{(1)}\right)+\lambda_{\mathrm{KL}} \mathrm{KL}\left(P_{i} \| Q\right)\right),
$$

where $I$ is the number of samples, $\operatorname{KL}\left(P_{i} \| Q\right)$ represents the Kullback-Leibler divergence between the probability mass function $P_{i}$ and $Q$, and $\lambda_{\mathrm{KL}}$ has a real value. $P_{i}$ is the Bernoulli distribution whose parameter is the probability $p\left(x^{(1)}\right)$ estimated from the TF-discrimination-DNN in each sample. $Q$ is the Bernoulli distribution whose probability parameter $q$ is set previously. This objective function operates as regularization for $p\left(\boldsymbol{x}^{(1)}\right)$. It also prevents the estimated probability $p\left(\boldsymbol{x}^{(1)}\right)$ from moving away from $q . \lambda_{\mathrm{KL}}$ is equivalent to the regularization parameter.

\subsection{Objective function for DOA estimation}

We define the objective function of the DOA estimation as $J_{\mathrm{DOA}}\left(\boldsymbol{x}^{(1)}\right)$. In addition to the cross-entropy function $J_{\mathrm{CE}}\left(\boldsymbol{x}^{(1)}\right)$, we use the angular error between a estimated DOA and the true DOA. The DOA is estimated as the weighted average, which is expressed as

$$
\bar{\theta}=\arg \left[\sum_{m=1}^{M} x_{m}^{(L)} \exp \left(-j \frac{2(m-1)}{M} \pi\right)\right],
$$

where $\arg [\cdot]$ indicates the argument of a complex value and $\exp \left(-j \frac{2(m-1)}{M} \pi\right)$ expresses the vector whose angle is the $m$ th label. The distance between the mean DOA $\bar{\theta}$ and the true DOA $\theta$ is expressed as

$$
J_{\text {angle }}(\bar{\theta}, \theta)=1-\cos (\bar{\theta}-\theta) .
$$

The cosine function is used to consider the periodicity of the direction. We transformed the cosine function to allow $J_{\text {angle }}(\bar{\theta}, \theta)$ to be 0 if $\bar{\theta}=\theta$. In this study, we use $J_{\text {angle }}+J_{\mathrm{CE}}$ as $J_{\mathrm{DOA}}\left(\boldsymbol{x}^{(1)}\right)$.

\section{Simulations and real-environment experiments}

The proposed method is validated by simulations and real environment experiments. Signals for training the DNN were generated by the simulations. The impulse response in a reverberant room was generated by the image method, and the performance of the trained model was validated with the 
simulated signal and the observed signals in real environments.

The experimental conditions include a sampling rate of $16 \mathrm{kHz}$ and a frame length of the short-time Fourier transform (STFT) of 256 points. The shift length of the STFT was 128 points. The microphone array was a uniform circular array comprising six microphones. The radius of the microphone array was $6 \mathrm{~cm}$.

GCC-PHAT [9], which was calculated for all microphone pairs, was used for the input feature of the DNN. GCC-PHAT was calculated from the target frame to seven frames earlier. The number of intermediate layers of the DOA estimationDNN was three. The number of units of the intermediate layers was 200. The output DOA labels were assigned in $5^{\circ}$ steps. The architecture shown in Fig. 1, it is called "Prop. Inter.," and the number of intermediate layers of the TF discrimination DNN was one. The architecture shown in Fig. 2, it is called "Prop. Sep.," and the number of intermediate layers of the TF discrimination DNN was three. The number of units of intermediate layers was 200. The activation function of the intermediate layers was the ReLU. We used Adam to optimize the DNN. The number of epochs was 30. Note that the configurations of the DNN architecture and learning were not optimized. The investigation of the DNN configuration is our future work.

The parameter $\lambda_{\mathrm{p}}$ was decreased with increasing epoch number as $\lambda_{\mathrm{p}}=\lambda_{\mathrm{p}}^{\text {init }} /($ epoch $)$ to learn the TF discrimination DNN in the first step, where (epoch) is the number of epochs. In the early epoch of learning, $J_{\mathrm{DOA}}$ tends to exhibit a large value. Consequently, when $\lambda_{\mathrm{p}}$ is set a small value in the entire epochs of learning, the TF discrimination DNN outputs 0 for all inputs. On the other hand, if $\lambda_{\mathrm{p}}$ is set to a large value in the entire epoch of learning, the TF discrimination DNN outputs 1 for all inputs. Therefore, to learn the TF discrimination adequately, we made $\lambda_{\mathrm{p}}$ decrease with increasing epoch number. $\lambda_{\mathrm{p}}^{\text {init }}$ may affect the performance of TF discrimination. Therefore, this effect of $\lambda_{\mathrm{p}}^{\text {init }}$ is discussed on the basis of the simulation experiments. $\lambda_{\mathrm{KL}}$ was 0.1 .

We now explain the conditions for the generation of the training data. The reverberation time $R T_{60}$ was set randomly from $0.08 \mathrm{~s}$ to $0.65 \mathrm{~s}$. White noise was added to the observed signal. The SNR of white-noise was determined randomly between $6 \mathrm{~dB}$ and $30 \mathrm{~dB}$. The distance between the sound source and the microphone array was set randomly between $0.7 \mathrm{~m}$ and $4.0 \mathrm{~m}$. The sound sources were 50 speech voices of a male or a female of 3-5 s duration.

The performance was evaluated by comparing the estimation accuracy of the conventional DOA estimation DNN with that of the estimated DOA for which the TF discrimination of the proposed DNN was classified as correct. The mean absolute error (MAE) was used for the evaluation of the DOA estimation accuracy.

The performance of TF discrimination of the proposed method was also evaluated. The ground truth of the TF discrimination DNN was determined from the DNN estimation outputs. If the estimated DOA was correct and the TF discrimination DNN output was 1, or the estimated DOA was incorrect and the TF discrimination DNN output was 0 , we assumed that the TF discrimination DNN estimated the

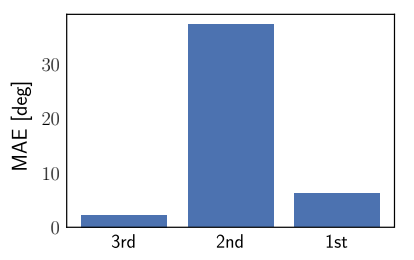

Fig. 3 The MAEs for each intermediate layer. The ordinal number is layer index from input layers.

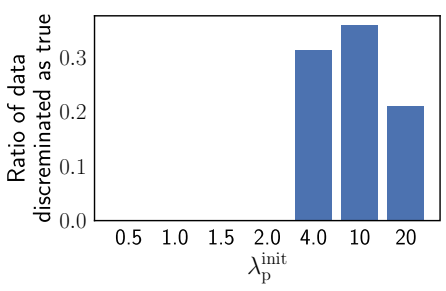

Fig. $4 \lambda_{\mathrm{p}}^{\text {init }}$ vs. the ratio of 1 of TF-discrimination-DNN. erroneous DOA correctly. The performance of TF discrimination was evaluated in terms of the accuracy, the precision and the recall.

4.1. Evaluation for TF discrimination DNN location of Prop. Inter.

We evaluated the TF discrimination DNN location for DOA estimation DNN in the Prop. Inter. architecture. In this experiment, the TF discrimination DNN architecture was not changed, but the input features were replaced with different intermediate-layer outputs of the DOA estimation DNN. $\lambda_{\mathrm{p}}^{\text {init }}$ was 4 . In this experiment, the DOA estimation was conducted in each frame. The results were evaluated in term of the MAEs of the proposed method. The test data conditions are described in the next section. The MAEs were calculated for each intermediate layer.

Figure 3 shows the MAEs for each intermediate layer. These results indicate that the intermediate feature of the layer the before output layer, which is the third intermediate layer had high accuracy. Therefore, we used the third intermediate layer for the TF discrimination DNN.

4.2. Evaluation of effect of the coefficient $\lambda_{\mathrm{p}}^{\text {init }}$

We examined the effect of the constant value $\lambda_{\mathrm{p}}^{\text {init }}$. The model of Prop. Inter. architecture was trained with each $\lambda_{\mathrm{p}}^{\text {init }}$, where the values of $\lambda_{\mathrm{p}}^{\text {init }}$ were $0.5,1.0,1.5,2.0,4.0,10.0$ and 20.0. The ratio of the number of label 1 to 0 of TFdiscrimination-DNN output for the test data was confirmed. The results are shown in Fig. 4. For $\lambda_{\mathrm{p}}^{\text {init }} \leq 2$, the TFdiscrimination-DNN does not output label 1 totally. Therefore, $\lambda_{\mathrm{p}}^{\text {init }}$ is set as 4 later hereafter.

4.3. Evaluation for simulation data

The test data were generated under different conditions for training. The reverberant times $R T_{60}$ were $0.10 \mathrm{~s}$ and $0.40 \mathrm{~s}$. The SNR of the white noise were fixed at $10 \mathrm{~dB}, 20 \mathrm{~dB}$ and $30 \mathrm{~dB}$. The distance between a sound source and the microphone array was set randomly between $0.7 \mathrm{~m}$ and $4.0 \mathrm{~m}$. The sound sources were 50 speech voices of 3-5 s duration for a male and a female, who were different from the training phase.

The results of the evaluation of DOA estimation are shown in Table 1. Our proposed method outperformed the conventional method for both architectures. The MAEs of the proposed method were at the same level as the SNR. For long reverberation times, the TF discrimination accuracy of the proposed method were degraded.

Tables 2, 3 and 4 show the accuracy, precision and recall for the estimation of TF-discrimination. The recall and precision are in trade-off relationship. In constant, the precision is directly linked to the estimation accuracy in our 
Table 1 MAE of DOA estimation in the simulation. Horizontal elements indicate the SNR, vertical elements indicate the reverberation time.

\begin{tabular}{|c|c|c|c|c|c|c|c|}
\hline Conv. & $30 \mathrm{~dB}$ & $20 \mathrm{~dB}$ & $10 \mathrm{~dB}$ & $\begin{array}{l}\text { Prop. } \\
\text { Inter. }\end{array}$ & $30 \mathrm{~dB}$ & $20 \mathrm{~dB}$ & $10 \mathrm{~dB}$ \\
\hline $0.10 \mathrm{~s}$ & 9.7 & 14.8 & 16.4 & $0.10 \mathrm{~s}$ & 1.0 & 1.3 & 1.2 \\
\hline $0.40 \mathrm{~s}$ & 17.1 & 19.8 & 19.8 & $0.40 \mathrm{~s}$ & 6.3 & 5.6 & 5.5 \\
\hline $\begin{array}{l}\text { Prop. } \\
\text { Sep. }\end{array}$ & $30 \mathrm{~dB}$ & $20 \mathrm{~dB}$ & $10 \mathrm{~dB}$ & & & & \\
\hline $0.10 \mathrm{~s}$ & 1.1 & 1.2 & 1.5 & & & & \\
\hline $0.40 \mathrm{~s}$ & 6.8 & 5.2 & 6.0 & & & & \\
\hline
\end{tabular}

Table 2 Accuracy of TF discrimination [\%].

\begin{tabular}{lccc|lccc}
\hline $\begin{array}{l}\text { Prop. } \\
\text { Inter. }\end{array}$ & $30 \mathrm{~dB}$ & $20 \mathrm{~dB}$ & $10 \mathrm{~dB}$ & $\begin{array}{l}\text { Prop. } \\
\text { Sep. }\end{array}$ & $30 \mathrm{~dB}$ & $20 \mathrm{~dB}$ & $10 \mathrm{~dB}$ \\
\hline $0.10 \mathrm{~s}$ & 87.5 & 86.5 & 81.9 & $0.10 \mathrm{~s}$ & 86.7 & 87.0 & 82.0 \\
$0.40 \mathrm{~s}$ & 87.0 & 83.9 & 82.5 & $0.40 \mathrm{~s}$ & 88.1 & 83.6 & 81.6 \\
\hline
\end{tabular}

Table 3 Precision of TF discrimination [\%].

\begin{tabular}{lccc|lccc}
\hline $\begin{array}{l}\text { Prop. } \\
\text { Inter. }\end{array}$ & $30 \mathrm{~dB}$ & $20 \mathrm{~dB}$ & $10 \mathrm{~dB}$ & $\begin{array}{l}\text { Prop. } \\
\text { Sep. }\end{array}$ & $30 \mathrm{~dB}$ & $20 \mathrm{~dB}$ & $10 \mathrm{~dB}$ \\
\hline $0.10 \mathrm{~s}$ & 98.9 & 98.0 & 97.2 & $0.10 \mathrm{~s}$ & 96.9 & 95.8 & 93.5 \\
$0.40 \mathrm{~s}$ & 97.3 & 95.8 & 93.6 & $0.40 \mathrm{~s}$ & 91.6 & 88.1 & 84.0 \\
\hline
\end{tabular}

Table 4 Recall of TF discrimination [\%].

\begin{tabular}{lccc|lccc}
\hline $\begin{array}{l}\text { Prop. } \\
\text { Inter. }\end{array}$ & $30 \mathrm{~dB}$ & $20 \mathrm{~dB}$ & $10 \mathrm{~dB}$ & $\begin{array}{l}\text { Prop. } \\
\text { Sep. }\end{array}$ & $30 \mathrm{~dB}$ & $20 \mathrm{~dB}$ & $10 \mathrm{~dB}$ \\
\hline $0.10 \mathrm{~s}$ & 79.0 & 76.3 & 65.7 & $0.10 \mathrm{~s}$ & 81.7 & 81.2 & 70.2 \\
$0.40 \mathrm{~s}$ & 78.3 & 72.8 & 64.9 & $0.40 \mathrm{~s}$ & 87.4 & 81.1 & 74.7 \\
\hline
\end{tabular}

proposed method. Therefore, appropriate results were obtained for improving DOA estimation accuracy.

4.4. Experiments in the real environments

We also evaluated the proposed method with real environment experiments. In these experiments, the DOA was estimated for every speech sentence. First, the DOAs were estimated for every frame in the speech sentence. By aggregating the estimated DOAs, we regard the most frequently estimated DOA as the estimated DOA of a speech sentence. In the proposed method, the only DOAs classified as correct are the aggregated ones.

The signals were observed in reverberant rooms with reverberant times $R T_{60}$ of $0.35 \mathrm{~s}$ and $1.05 \mathrm{~s}$. White noise was added under the same conditions as in the simulation experiments. The distance between the microphone array and a sound source was $2.0 \mathrm{~m}$ in the room of $R T_{60}=0.35 \mathrm{~s}$ and it was $1.2 \mathrm{~m}$ or $2.0 \mathrm{~m}$ in the room of $R T_{60}=1.05 \mathrm{~s}$. The results are shown in Table 5. The proposed method also improved the estimation accuracy for real environments. The improvements in the Prop. Sep. architecture were smaller than those for the simulation experiments. In particular, when $R T_{60}$ was $1.05 \mathrm{~s}$, the distance was $2.0 \mathrm{~m}$ and the SNR was $10 \mathrm{~dB}$, the accuracy did not improve. Meanwhile, Prop. Inter. improved the accuracy more than Prop. Sep. The reason why this result was obtained may be that the Prop. Inter. architecture is similar to the multitask learning model [10]. It is empirically known that multitask learning can give better performance than single task learning. From the perspective of multitask learning, this results indicates that intermediate feature for the DOA estimation is also useful for the TF discrimination.
Table 5 MAE of DOA estimation in real environment.

Horizontal elements indicate the SNR, vertical elements indicate the reverberation time and the distance.

\begin{tabular}{|c|c|c|c|c|c|c|c|}
\hline Conv. & $30 \mathrm{~dB}$ & $20 \mathrm{~dB}$ & $10 \mathrm{~dB}$ & $\begin{array}{l}\text { Prop. } \\
\text { Sep. }\end{array}$ & $30 \mathrm{~dB}$ & $20 \mathrm{~dB}$ & $10 \mathrm{~dB}$ \\
\hline $\begin{array}{l}0.35 \mathrm{~s}, \\
2.0 \mathrm{~m}\end{array}$ & 0.7 & 0.9 & 1.1 & $\begin{array}{l}0.35 \mathrm{~s}, \\
2.0 \mathrm{~m}\end{array}$ & 0.7 & 0.8 & 1.0 \\
\hline $\begin{array}{l}1.05 \mathrm{~s}, \\
1.2 \mathrm{~m}\end{array}$ & 4.3 & 4.8 & 4.5 & $\begin{array}{l}1.05 \mathrm{~s}, \\
1.2 \mathrm{~m}\end{array}$ & 3.5 & 3.6 & 3.6 \\
\hline $\begin{array}{l}1.05 \mathrm{~s}, \\
2.0 \mathrm{~m}\end{array}$ & 8.6 & 5.8 & 5.8 & $\begin{array}{l}1.05 \mathrm{~s} \\
2.0 \mathrm{~m}\end{array}$ & 5.3 & 5.6 & 5.8 \\
\hline $\begin{array}{l}\text { Prop. } \\
\text { Inter. }\end{array}$ & $30 \mathrm{~dB}$ & $20 \mathrm{~dB}$ & $10 \mathrm{~dB}$ & & & & \\
\hline $\begin{array}{l}0.35 \mathrm{~s}, \\
2.0 \mathrm{~m}\end{array}$ & 0.6 & 0.8 & 0.9 & & & & \\
\hline $\begin{array}{l}1.05 \mathrm{~s}, \\
1.2 \mathrm{~m}\end{array}$ & 2.5 & 2.7 & 2.9 & & & & \\
\hline $\begin{array}{l}1.05 \mathrm{~s}, \\
2.0 \mathrm{~m}\end{array}$ & 3.6 & 3.7 & 4.4 & & & & \\
\hline
\end{tabular}

\section{Conclusion and future works}

To improve the accuracy of DOA estimation, we proposed a discrimination method to classify the estimated DOA as correct or an error. This discrimination was learned from the objective function of the DOA estimation as a binary classification. The model learned from the simulation data could discriminate the estimated DOA. In real environments, the estimation accuracy was improved to more than that for a conventional DNN method by estimating the DOA using only the output discriminated to be correct. Future work will involve the investigation of the DNN architecture for TF discrimination.

\section{References}

[1] Y. LeCun, Y. Bengio and G. Hinton, "Deep learning," Nature, 521, 436-444 (2015).

[2] S. Araki, H. Sawada, R. Mukai and S. Makino, "DOA estimation for multiple sparse sources with normalized observation vector clustering," Proc. ICASSP 2006, Vol. 5, pp. 33-36 (2006).

[3] X. Li, S. Yan, X. Ma and C. Hou, "Spherical harmonics music versus conventional music," Appl. Acoust., 72, 646-652 (2011).

[4] V. Nair and G. E. Hinton, "Rectified linear units improve restricted Boltzmann machines," Proc. ICML-10, pp. 807-814 (2010).

[5] X. Xiao, S. Zhao, X. Zhong, D. L. Jones, E. S. Chng and H. Li, "A learning-based approach to direction of arrival estimation in noisy and reverberant environments," Proc. ICASSP 2015, pp. 2814-2818 (2015).

[6] R. Takeda and K. Komatani, "Sound source localization based on deep neural networks with directional activate function exploiting phase information," Proc. ICASSP 2016, pp. 405409 (2016).

[7] S. Chakrabarty and E. A. P. Habets, "Broadband DOA estimation using convolutional neural networks trained with noise signals," arXiv preprint arXiv:1705.00919 (2017).

[8] C. M. Bishop, Pattern Recognition and Machine Learning (Springer, 2006).

[9] K. Charles and C. Glifford, "The generalized correlation method for estimation of time delay," IEEE Trans. Acoust. Speech Signal Process., 24, 320-327 (1976).

[10] R. Collobert and J. Weston, “A unified architecture for natural language processing: Deep neural networks with multitask learning," Proc. 25th ICML, pp. 160-167 (2008). 